\title{
Life-threatening gastrointestinal bleeding during targeted therapy for advanced renal cell carcinoma: a case report
}

\author{
Shintaro Fujihara ${ }^{1 *}$, Hirohito Mori ${ }^{1}$, Hideki Kobara ${ }^{1}$, Takehiro Suenaga ${ }^{2}$, Yuji Hayashida², Mikio Sugimoto², \\ Yoshiyuki Kakehi ${ }^{2}$ and Tsutomu Masaki ${ }^{1}$
}

\begin{abstract}
Background: Temsirolimus has important clinical activity in both untreated and previously treated patients with advanced renal cell carcinoma. Targeted therapy-related stomatitis and mucositis have occurred during targeted therapies, but there is no consensus on which strategy is the most effective. We herein report a case in which several sessions of endoscopic hemostasis with argon plasma coagulation (APC) effectively resolved life-threatening gastrointestinal bleeding that had occurred during targeted therapy. This is the first case report of such an adverse drug reaction in the literature.
\end{abstract}

Case presentation: A 47-year-old female patient with advanced renal cell carcinoma was treated with temsirolimus. Eight weeks after starting targeted therapy, the patient was admitted to our hospital for worsened fatigue, pallor, and hematemesis. A complete blood count showed a marked drop in her hemoglobin level from $10.1 \mathrm{~g} / \mathrm{dll} 4$ days earlier to $2.9 \mathrm{~g} / \mathrm{dl}$. Esophagogastroduodenoscopy revealed diffuse mucosal bleeding of the antrum. Endoscopy revealed diffuse reddish spots that resembled gastric antral vascular ectasia (GAVE) extending from the pylorus into the antrum. One month after endoscopic hemostasis with APC and stopping temsirolimus, significant improvement was shown in the gastric erythema and GAVE like lesions.

Conclusion: Minor hemorrhagic events are relatively common in patients treated with targeted agents. Lifethreatening hemorrhagic events are rarer than minor hemorrhagic complications. In the present case, endoscopic hemostasis with APC effectively prevented severe anemia and blood loss due to gastrointestinal bleeding.

Keywords: Gastrointestinal bleeding, Temsirolimus, Gastric antral vascular ectasia, Targeted therapy, Argon plasma coagulation

\section{Background}

With the advent of targeted agents for the treatment of renal cell carcinoma (RCC), most patients are being treated continuously for increasingly long periods of time. This has raised new challenges related to management of the associated adverse events [1].

Temsirolimus has important clinical activity in both untreated and previously treated patients with advanced RCC [2]. Severe adverse events associated with temsirolimus are uncommon; however, the most frequent grade 3 or 4

\footnotetext{
*Correspondence: joshin@med.kagawa-u.ac.jp

'Department of Gastroenterology and Neurology, Kagawa University Faculty of Medicine/Graduate School of Medicine, 1750-1 Ikenobe, Miki-cho, Kita-gun, Kagawa Prefecture 761-0793, Japan

Full list of author information is available at the end of the article
}

adverse events are anemia, asthenia, and hyperglycemia [3]. Targeted therapy-related stomatitis and mucositis have occurred during targeted therapy, but there is no consensus on which strategy is the most effective.

We herein report a case in which several sessions of endoscopic hemostasis with argon plasma coagulation (APC) effectively resolved life-threatening gastrointestinal bleeding that had occurred during targeted therapy.

\section{Case presentation}

The patient was a 40-year-old woman diagnosed as having left renal cell carcinoma (cT3aN2M1) and adrenal gland metastasis. She was surgically treatment, having radical left nephrectomy in October 2009. There was no

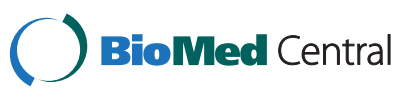


history of chronic kidney disease, valvular heart diseases, liver cirrhosis and other disorders. The resected tumor was pathologically diagnosed as collecting duct carcinoma, G3 INF $\beta, \mathrm{v}(+), \operatorname{ly}(+) 7 \mathrm{~cm}$ pT2. In April 2010, follow-up computerized tomography (CT) revealed newly bronchial lymph node metastasis, and she received initially 30 Gy radiation therapy for lymph node metastasis to prevent bronchial stenosis. After the radiation therapy, she complained of epigastralgia, so we performed esophagogastroduodenoscopy (EGD). However it revealed no abnormality (Figure 1).

Temsirolimus (15 mg/day) was started in June 2012. Her blood test results at various follow-ups showed mild stable anemia (hemoglobin of 10.1-10.7 g/dl), but other laboratory parameters were normal on all occasions, including her chemistry panel and liver function tests.

Eight weeks after starting targeted therapy, the patient was admitted to the Department of Gastroenterology for worsening fatigue, pallor, and hematemesis. A complete blood count showed a marked drop in her hemoglobin level from $10.1 \mathrm{~g} / \mathrm{dl} 4$ days earlier to $2.9 \mathrm{~g} / \mathrm{dl}$. Her prothrombin time and activated partial thrombin time were normal. She had made no dosage changes to any of her medications, and had not started/stopped any medication during targeted therapy.

EGD revealed diffuse mucosal bleeding of the antrum (Figure 2A). Endoscopy showed diffuse reddish spots that resembled gastric antral vascular ectasia (GAVE) extending from the pylorus into the antrum. Highresolution magnifying endoscopy (ME) revealed that the mucosa was friable, and oozing bleeding occurred from ectatic vessel rupture (Figure 2B). In the background mucosa, brownish subepithelial capillaries were clearly visualized by ME with narrow-band imaging, and

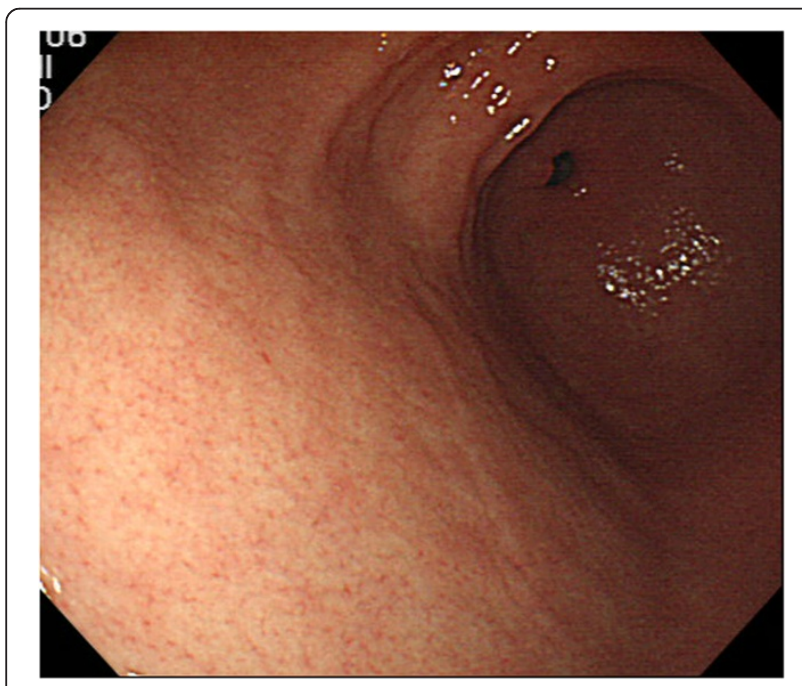

Figure 1 Previous esophagogastroduodenoscopy before starting targeted therapy. congestive and dilated subepithelial capillaries were seen in the background mucosa (Figure 2C). These findings represented a major change from the EGD performed before targeted therapy. Pathologic examination revealed interstitial fibrosis and extensive edema with capillary and venous dilatation in the submucosa extending into the mucosa.

This complication was most likely an adverse reaction to temsirolimus, because the patient had no underlying medical conditions associated with GAVE and had no evidence of GAVE on EGD before starting temsirolimus.

Treatment with a proton-pump inhibitor was started, and follow-up with repeat endoscopic hemostasis with argon plasma coagulation (APC) was performed once to twice weekly (Additional file 1). The patient required four to six units of packed red blood cells every day, and the need for blood transfusion gradually decreased with the hemostasis treatment. After three sessions of APC, the patient did not need the blood transfusions, and the improvement of GAVE like lesions.

Overall, the patient required a total of four sessions of endoscopic hemostasis and 38 units of transfused blood for severe anemia during these 2 weeks.

Four weeks after discontinuing temsirolimus, the patient's symptoms resolved and she was discharged in good clinical condition 60 days after hospital admission. One month after hospital discharge, significant improvement was seen in the gastric erythema and GAVE like lesions (Figure 3).

\section{Discussion}

Six targeted agents for the treatment of advanced RCC are now approved and in clinical use: the tyrosine kinase inhibitors sunitinib and pazopanib, the multikinase inhibitor sorafenib, the anti-vascular endothelial growth factor monoclonal antibody bevacizumab, and the mammalian target of rapamycin (mTOR) inhibitors temsirolimus and everolimus [1].

Temsirolimus is an inhibitor of mTOR kinase, a component of intracellular signaling pathways involved in the growth and proliferation of cells $[4,5]$ and the response of such cells to hypoxic stress [6]. The inhibition of angiogenesis by temsirolimus is clinically relevant because unregulated angiogenesis is prominent in RCC [7].

Minor hemorrhagic events are relatively common in patients treated with targeted agents; the most common event reported in patients treated with bevacizumab, sunitinib, temsirolimus, and everolimus is epistaxis, which usually resolves without medical attention. Lifethreatening hemorrhagic events are rarer than minor hemorrhagic complications. In the case of bevacizumab, serious hemorrhage appears to be more frequently associated with specific tumor types, such as non-small cell lung cancer or cancer of the gastrointestinal tract [8]. 

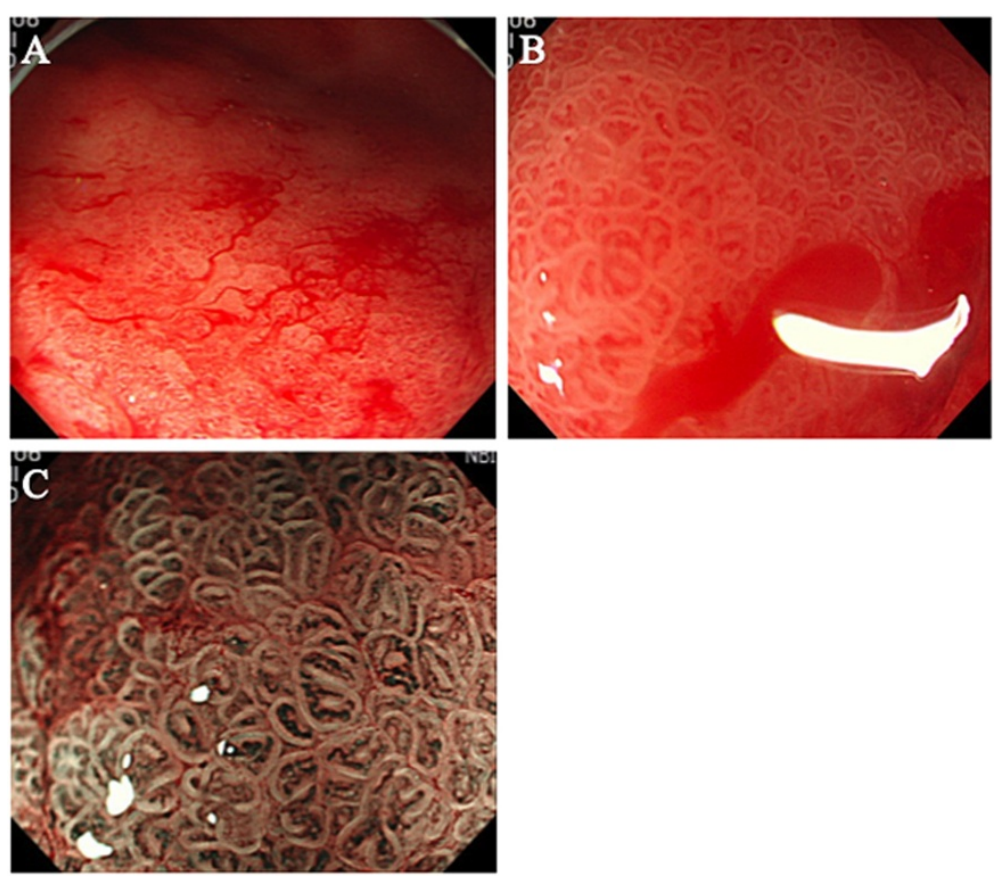

Figure 2 Esophagogastroduodenoscopy (EGD) when the patient presented with gastrointestinal bleeding. (A) Esophagogastroduodenoscopy revealed diffuse mucosal bleeding of the antrum that resembled gastric antral vascular ectasia. (B) Magnifying endoscopy (ME) findings. The gastric mucosa was friable, and oozing bleeding occurred from ectatic vessel rupture. (C) ME with narrow-band imaging revealed congestive and dilated subepithelial capillaries in the background mucosa.

GAVE, also known as watermelon stomach, is a rare cause of upper gastrointestinal bleeding that is often confused with portal hypertensive gastropathy, both of which can occur in patients with cirrhosis [9]. A clear relationship between mTOR inhibitors and the pathogenesis of

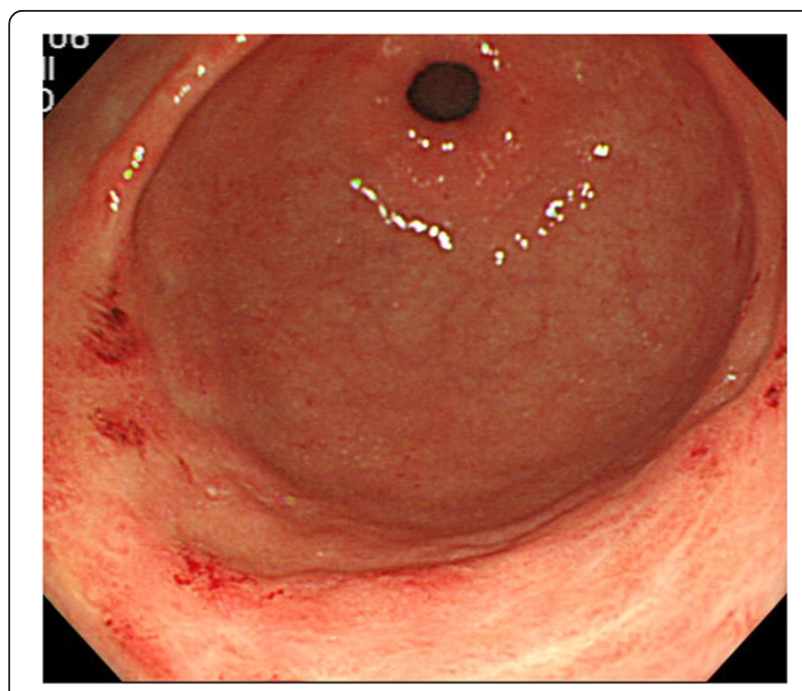

Figure 3 Esophagogastroduodenoscopy showed resolution of the patient's gastric antral vascular ectasia 1 month after stopping temsirolimus, but diffuse angiodysplasia at the antrum was present. targeted therapy-related stomatitis and GAVE has not been shown. In one case, a patient with gastrointestinal stromal tumors showed GAVE 8 months after starting imatinib [10]. Recently, a paper by Kim et al. [11] provided information on the activation of mTOR signaling pathways that promote wound healing in the stomach. Inhibitors of mTOR kinase may affect mucosal healing in the stomach and trigger gastritis and gastrointestinal bleeding.

Endoscopic therapy remains the treatment of choice for GAVE. Photocoagulation using a neodymium: yttrium-aluminum-garnet laser and APC have been successful in treating GAVE and abolishing or reducing transfusion requirements $[12,13]$. In the present case, several sessions of endoscopic hemostasis with APC effectively prevented severe anemia and blood loss due to gastrointestinal bleeding.

\section{Conclusion}

Minor hemorrhagic events are relatively common in patients treated with targeted agents. Targeted therapyrelated stomatitis and mucositis have occurred during targeted therapies, but there is no consensus on which strategy is the most effective. mTOR inhibitors may be related to the development of stomatitis, mucositis, and vascular ectasia through mTOR pathways. In the present case, endoscopic hemostasis with APC effectively prevented 
severe anemia and blood loss due to GAVE. This is the first case report of such an adverse drug reaction in the literature.

\section{Consent}

Written informed consent was obtained from the patient for publication of this Case report and any accompanying images. A copy of the written consent is available for review by the Editor of this journal.

\section{Additional file}

Additional file 1: Endoscopic hemostasis with argon plasma coagulation (APC).

\section{Competing interests}

The authors declare that they have no competing interests.

\section{Authors' contributions}

SF, HM, HK, TS, YH, MS and YK have made substantial contributions to acquisition of data and interpretation data. TM has given final approval of the version to be published. All authors read and approved the final manuscript.

\section{Acknowledgements}

We thank Dr. Noriko Nishiyama and Dr. Makoto Oryu for providing technical and editorial assistance.

\section{Author details}

${ }^{1}$ Department of Gastroenterology and Neurology, Kagawa University Faculty of Medicine/Graduate School of Medicine, 1750-1 Ikenobe, Miki-cho, Kita-gun, Kagawa Prefecture 761-0793, Japan. ${ }^{2}$ Department of Urology, Kagawa University, Faculty of Medicine, 1750-1 Ikenobe, Miki-cho, Kita-gun, Kagawa Prefecture 761-0793, Japan.

Received: 3 December 2012 Accepted: 8 July 2013

Published: 10 July 2013

\section{References}

1. Tim E, Sternberg CN, et al: Targeted therapies for renal cell carcinoma: review of adverse event management strategies. J Natl Cancer Inst 2012, 104:93-113.

2. Atkins $M B$, Hidalgo $M$, Stadler $W M$, et al: Randomized phase II study of multiple dose levels of CCl-779, a novel mammalian target of rapamycin kinase inhibitor, in patients with advanced refractory renal cell carcinoma. J Clin Oncol 2004, 22(5):909-918.

3. Hudes G, Carducci M, Tomczak P, et al: Temsirolimus, interferon alfa, or both for advanced renal-cell carcinoma. N Engl J Med 2007, 356(22):2271.

4. Schmelzle T, Hall MN: TOR, a central controller of cell growth. Cell 2000 103:253-262.

5. Fingar DC, Richardson CJ, Tee AR, Cheatham L, Tsou C, Blenis J: mTOR controls cell cycle progression through its cell growth effectors S6K1 and 4E-BP1 /eukaryotic translation factor 4E. Mol Cell Biol 2004, 24:200-216.

6. Hudson CC, Liu M, Chiang GG, et al: Regulation of hypoxia-inducible factor $1 a$ expression and function by the mammalian target of rapamycin. Mol Cell Biol 2002, 22:7004-7014

7. Pantuck AJ, Zeng G, Belldegrun AS, Figlin RA: Pathobiology, prognosis, and targeted therapy for renal cell carcinoma: exploiting the hypoxiainduced pathway. Clin Cancer Res 2003, 9:4641-4652.

8. EU SmPC Avastin. http://www.emea.europa.eu/ema/index.jsp?curl=pages/ medicines/human/medicines/000582/human_med_000663. jsp\&mid=WC0b01ac058001d124. Accessed July 12, 2013.

9. Payen $J \mathrm{~L}$, Calès $P$, Voigt JJ, et al: Severe portal hypertensive gastropathy and antral vascular ectasia are distinct entities in patients with cirrhosis. Gastroenterology 1995, 108(1):138-144.
10. Ehab Saad A, Fadi M, Arafat T: Gastric antral vascular ectasia in a patient with GIST after treatment with imatinib: case report and literature review. Jpn J Clin Oncol 2012, 42(5):447-450.

11. Kim YW, Lee $W H$, Choi SM, et al: DA6034 promotes gastric epithelial cell migration and wound-healing through the mTOR pathway. J Gastroenterol Hepatol 2012, 27:397-405.

12. Gostout CJ, Viggiano TR, Ahlquist DA, Wang KK, Larson MV, Balm R: The clinical and endoscopic spectrum of the watermelon stomach. J Clin Gastroenterol 1992, 15:256-263.

13. Kwan V, Bourke MJ, Williams SJ, et al: Argon plasma coagulation in the management of symptomatic gastrointestinal vascular lesions: experience in 100 consecutive patients with long-term follow-up. Am $J$ Gastroenterol 2006, 101:58-63.

doi:10.1186/1471-2369-14-141

Cite this article as: Fujihara et al:: Life-threatening gastrointestinal bleeding during targeted therapy for advanced renal cell carcinoma: a case report. BMC Nephrology 2013 14:141.

\section{Submit your next manuscript to BioMed Central and take full advantage of:}

- Convenient online submission

- Thorough peer review

- No space constraints or color figure charges

- Immediate publication on acceptance

- Inclusion in PubMed, CAS, Scopus and Google Scholar

- Research which is freely available for redistribution 